\title{
Habilidades funcionais da criança com Síndrome Congênita pelo Zika Vírus e sobrecarga da mãe
}

\author{
Functional skills of children with Congenital Zika Virus \\ Syndrome and overload of mothers
}

\section{Habilidades funcionales de niños con Síndrome Congénito del Virus Zika y sobrecarga de madres}

\author{
Marinna Coelho Oliveira ${ }^{1}$, Gabriela Eiras Ortoni ${ }^{2}$, Thereza Cristina \\ Rodrigues Abdalla Veríssimo ${ }^{3}$, Maysa Ferreira Martins Ribeiro ${ }^{4}$, Andréa \\ Souza Rocha ${ }^{5}$, Francine Aguilera Rodrigues da Silva6, Mônica Isabella \\ Chagas Moreira ${ }^{7}$, Mayara Cordeiro de Faria ${ }^{1}$, Cejane Oliveira Martins \\ Prudente $^{8}$, Paulo Fernando Lôbo Corrêa ${ }^{9}$
}

\footnotetext{
1.Fisioterapeuta, residente em Saúde Funcional e Reabilitação. Centro Estadual de Reabilitação e Readaptação Dr. Henrique Santillo - CRER, Goiânia-GO, Brasil.

2.Enfermeira, especialista em Enfermagem em Emergência pela Faculdade Estácio de Sá, Goiânia-GO, Brasil.

3.Fisioterapeuta, mestre pela Pontifícia Universidade Católica de Goiás - PUC-Goiás, Goiânia-GO, Brasil. 4.Fisioterapeuta, pós-doutora pela Universidade Federal de Goiás - UFG, docente Universidade Estadual de Goiás - UEG e da PUC-Goiás, Goiânia-GO, Brasil.

5.Fisioterapeuta, mestre pela UFG, docente de pós-graduação, Goiânia-GO, Brasil.

6.Fisioterapeuta, mestre pela UFG-GO, Goiânia-GO, Brasil.

7.Fisioterapeuta, mestre pela PUC-GO, Goiânia-GO, Brasil.

8.Fisioterapeuta, pós-doutora pela UFG, docente da PUC-Goiás e da UEG, Goiânia-GO, Brasil.

9.Fisioterapeuta, mestre pela UFG, Goiânia-GO, Brasil.
}

\begin{abstract}
Resumo
Introdução. A Síndrome Congênita pelo Zika Vírus (SCZV) provoca uma série de alterações encefálicas que tendem a impactar gravemente o desenvolvimento neuropsicomotor dessas crianças. Objetivo. Analisar a relação entre habilidades funcionais das crianças com SCZV e a sobrecarga de suas mães. Método. Estudo transversal analítico, realizado em um centro de reabilitação em Goiânia, Goiás, Brasil. Foi aplicado questionário semiestruturado com dados sociodemográficos das mães e clínicos da criança; Questionário de Avaliação da Sobrecarga do Cuidador Informal (QASCI); Pediatric Evaluation of Disability Inventory - Computer Adaptive Test (PEDI-CAT). Resultados. Amostra composta por 32 crianças com SCZV e suas mães. Todas as mães possuíam sobrecarga de moderada a elevada. Todas as crianças apresentavam atraso nas habilidades funcionais. Foram estabelecidas correlações moderadas entre os domínios do PEDI-CAT e os domínios do QASCI (reação às exigências, mecanismos de eficácia e controle e satisfação com o papel e com o familiar), bem como com a sua pontuação final. Conclusão. As habilidades funcionais dessas crianças são gravemente impactadas. Quanto pior as habilidades funcionais das crianças, menor a sobrecarga em reação às exigências e mecanismos de eficácia e controle e maior sobrecarga na satisfação com o papel e com o familiar.
\end{abstract}

Unitermos. Zika vírus; Microcefalia; Destreza motora; Esgotamento do cuidador; Carga de trabalho

\footnotetext{
Abstract

Introduction. Congenital Zika Virus Syndrome (SCZV) causes a series of brain changes that tend to severely impact the neuropsychomotor development of these children. Objective. To
} 
analyze the relationship between functional abilities of children with SCZV and their mothers' burden. Method. Cross-sectional analytical study, carried out in a rehabilitation center in Goiânia, Goiás, Brazil. A semi-structured questionnaire was applied with sociodemographic data of the mothers and the child's clinicians; Informal Caregiver Burden Assessment Questionnaire (QASCI); Pediatric Evaluation of Disability Inventory - Computer Adaptive Test (PEDI-CAT). Results. Sample consisting of 32 children with SCZV and their mothers. All mothers had moderate to high burden. All children had a delay in functional skills. Moderate correlations were established between the PEDI-CAT domains and the QASCI domains (reaction to demands, mechanisms of effectiveness and control and satisfaction with the role and the family member), as well as with their final score. Conclusion. The functional abilities of these children are severely impacted. The worse the children's functional abilities, the less the overload in response to demands and mechanisms of effectiveness and control and the greater overload in satisfaction with the role and the family member.

Keywords. Zika virus; Microcephaly; Motor dexterity; Exhaustion of the caregiver; Workload

\section{Resumen}

Introducción. El síndrome congénito del virus del Zika (SCZV) provoca una serie de cambios cerebrales que tienden a afectar gravemente el desarrollo neuropsicomotor de estos niños. Objetivo. Analizar la relación entre las capacidades funcionales de los niños con SCZV y la sobrecarga materna. Método. Estudio analítico transversal, realizado en un centro de rehabilitación en Goiânia, Goiás, Brasil. Se aplicó un cuestionario semiestructurado con datos sociodemográficos de las madres y médicos del niño; Cuestionario informal de evaluación de la carga del cuidador (QASCI); Inventario de evaluación de discapacidad pediátrica: pruebas informáticas adaptativas (PEDI-CAT). Resultados. Muestra compuesta por 32 niños con SCZV y sus madres. Todas las madres tenían una carga de moderada a alta. Todos los niños mostraron un retraso en las habilidades funcionales. Se establecieron correlaciones moderadas entre los dominios PEDI-CAT y los dominios QASCI (reacción a las demandas, mecanismos de efectividad y control y satisfacción con el rol y el familiar), así como con su puntuación final. Conclusión. Las capacidades funcionales de estos niños se ven gravemente afectadas. Cuanto peores son las capacidades funcionales del niño, menor es la carga de responder a las demandas y mecanismos de efectividad y control y mayor la carga de satisfacción con el rol y el familiar.

Palabras clave. Virus del Zika; Microcefalia; Destreza motora; Agotamiento del cuidador; Carga de trabajo

Trabalho realizado no Centro Estadual de Reabilitação e Readaptação Dr. Henrique Santillo - CRER, Goiânia-GO, Brasil.

\section{INTRODUÇÃO}

\section{A Síndrome Congênita do Zika Vírus (SCZV) provoca}

uma série de alterações encefálicas, sendo que as características de maior destaque são a presença de calcificações intracranianas, especialmente na junção da substância branca e cinzenta; ventriculomegalia e volume cerebral reduzido. No entanto, ela ainda está em 
investigação fenotípica ${ }^{1,2}$. A SCZV é acompanhada por sinais e sintomas variados decorrentes dessas características como microcefalia fetal ou pós-natal; hidrocefalia; epilepsia; irritabilidade; anormalidades visuais, auditivas, motoras e disfagia. Esses sinais e sintomas acometem as crianças de forma variada em sua frequência e intensidade. No entanto, o seu impacto no desenvolvimento neuropsicomotor tende a ser grave ${ }^{3}$.

O desenvolvimento neuropsicomotor das crianças com SCZV vem sendo estudado de forma crescente ${ }^{4}$. O desempenho motor tem sido classificado no mais alto nível de comprometimento, pois, em mais de $80 \%$ dos casos corresponde a classificação nível V do Gross Motor Function Classification System (GMFCS) ${ }^{1}$. O desempenho motor é relacionado de forma direta com as habilidades funcionais. Ou seja, pior desempenho motor representa declínio nas habilidades funcionais ocorrendo limitações nas atividades diárias e impactando de forma negativa na participação ${ }^{5}$.

O declínio das habilidades funcionais acaba por colocar as crianças com SCZV como dependentes da pessoa mais próxima para a realização das suas atividades diárias. Essa pessoa mais próxima é definida como cuidador principal. Geralmente, o cuidador principal da criança é a mãe. A literatura chega a apontar que em mais de $97 \%$ dos casos de crianças com SCZV a mãe era a cuidadora principal ${ }^{6}$.

O tempo despendido na assistência às crianças com declínio das habilidades funcionais é notoriamente extenso, devido às demandas de atenção em ambiente domiciliar e 
social, que tendem a aumentar conforme a criança cresce. Além disso, o declínio das habilidades funcionais presente em crianças com deficiências parece influenciar de forma diretamente proporcional na sobrecarga de suas mães ${ }^{7}$.

A sobrecarga das mães por vezes é negligenciada, visto que o maior alvo das pesquisas com SCZV visa a identificação dos aspectos relacionados à criança ${ }^{8}$. Portanto, torna-se importante analisar, também, a figura da mãe, pois, ela é a pessoa mais próxima à criança e aquela que atende as demandas de atenção em ambiente domiciliar e social. Por isso, o objetivo desse estudo relacionar as habilidades funcionais das crianças com SCZV com a sobrecarga das mães.

\section{MÉTODO}

\section{Amostra}

Trata-se de um estudo transversal analítico, realizado em um centro estadual de reabilitação de Goiânia - Goiás. Estudo realizado de março de 2019 a dezembro de 2020 e a coleta de dados entre maio e agosto de 2019. Foi aprovado pelo Comitê de Ética em Pesquisa da Pontifícia Universidade Católica de Goiás (Certificado de Apresentação de Apreciação Ética de número 10175419.6.0000.0037). O Termo de Consentimento Livre e Esclarecido (TCLE) como participante da pesquisa e responsável pela criança foi assinado por todas as mães, após receberem todos os esclarecimentos sobre a pesquisa. 
A amostra foi composta por mães de crianças com SCZV. As crianças com SCZV deveriam ter o diagnóstico confirmado por meio de exame laboratorial e/ou achados clínicos, além de estarem em acompanhamento com equipe multiprofissional e/ou médica da instituição. As crianças não poderiam ter outras doenças neurológicas que não tivessem relação com a SCZV. Os critérios de inclusão para as mães foram: idade superior a 18 anos e ser a cuidadora principal da criança. Os critérios de exclusão para as mães foram: incapaz de responder instruções verbais e recusa a participar da pesquisa.

\section{Procedimentos}

Os instrumentos utilizados para a coleta de dados foram: questionário semiestruturado sobre os dados sociodemográficos das mães e clínicos da criança, usado para caracterizar a amostra e elaborado pelos próprios pesquisadores; o Questionário de Avaliação da Sobrecarga do Cuidador Informal (QASCI) ${ }^{9}$, usado para analisar os domínios da sobrecarga física, emocional e social das mães; e o Pediatric Evaluation of Disability Inventory - Computer Adaptive Test (PEDI-CAT) ou Inventário de Avaliação Pediátrica de Incapacidade - Testagem Computadorizada Adaptativa ${ }^{10}$, usado para analisar as habilidades funcionais das crianças.

O questionário sociodemográfico investigou os seguintes dados referentes as mães: idade, estado civil, escolaridade, renda familiar, uso de transporte próprio ou 
não, residência própria, plano de saúde, se possuía algum benefício, se era submetida a tratamento psicológico, e o número de filhos.

Os seguintes dados referentes ao perfil clínico das crianças foram investigados: idade, sexo, idade gestacional ao nascimento, classificação da microcefalia, presença de crises convulsivas, uso de cadeira de rodas, se a criança andava, tratamento com equipe multiprofissional, presença de espasticidade, irritabilidade, alterações visuais e auditivas.

O QASCI foi desenvolvido em Portugal e validado no Brasil $^{9}$, é de fácil e rápida aplicação (cerca de vinte minutos). Avalia a sobrecarga física, emocional e social de cuidadores informais por meio de sete domínios: Sobrecarga emocional; Implicações na vida pessoal; Sobrecarga financeira; Reação às exigências; Mecanismos de eficácia e controle; Suporte familiar; Satisfação com o papel e com o familiar. Eles são classificados por meio de uma escala ordinal entre 1 a 5 . 0 QASCI avalia esses itens por meio da percepção subjetiva desses quesitos nas últimas quatro semanas, classificando a sobrecarga em baixa, moderada, elevada ou extrema11-13.

O PEDI-CAT foi desenvolvido nos Estados Unidos; traduzido, validado e adaptado culturalmente para o Brasil $^{10}$. Ele pode ser aplicado em crianças e jovens de 0 a 21 anos de idade, em todos os diagnósticos clínicos, condições e perfis de comunidades. O PEDI-CAT têm 276 itens divididos em quatro domínios, baseados em atividades funcionais: Atividades Diárias (68 itens), Mobilidade (97 itens), 
Social/Cognitivo (60 itens) e Responsabilidade (51 itens). Os três primeiros domínios são graduados em uma escala de 1 a 4, com 1 representando incapacidade para realizar a atividade e 4 atividade de fácil execução. A opção "não sei" consta em todos os itens ${ }^{14}$. Esses domínios podem ser aplicados em conjunto ou isoladamente. Nessa pesquisa 0 domínio Responsabilidade não foi coletado. A versão do PEDI-CAT utiliza um software que usa algoritmos direcionando perguntas precisas e individualizadas reduzindo o tempo de aplicação. O instrumento pode ser preenchido pelos cuidadores ou na presença de um profissional de saúde habilitado $^{10}$. O PEDI-CAT utiliza dois tipos de escores. O escore contínuo não considera a idade da criança e varia de 20 a 80. O escore normativo é representado pelo escore $T$, em que a média para cada faixa etária é de 50, e apresenta desvio padrão de 10. Assim, as pontuações variam entre 30 e $70^{15}$.

Foi feito contato com os autores responsáveis pela versão brasileira do QASCI e eles autorizaram o seu uso em cuidadores informais de crianças acometidas pelo vírus Zika. O uso do PEDI-CAT no Brasil é feito por meio da licença de uso do software, sendo que esta licença foi adquirida pelos pesquisadores para o período de seis meses.

A triagem das mães e crianças foi feita para a verificação dos critérios de inclusão e exclusão, utilizando informações contidas nos prontuários e coletadas com os profissionais responsáveis pelo atendimento das crianças. 
Posteriormente as mães elegíveis para o estudo foram esclarecidas sobre a pesquisa e convidadas a participar.

A coleta de dados iniciou-se com uma entrevista semiestruturada para coleta dos dados referentes ao perfil sociodemográfico e clínico das mães, e clínico das crianças. As informações referentes ao questionário sociodemográfico foram complementadas e conferidas no prontuário eletrônico da instituição. A análise dos prontuários foi feita após a autorização da instituição, mediante o Termo de Compromisso de Utilização de Dados e consentimento das mães. Em seguida eram aplicados o QASCI e o PEDI-CAT. Os itens do QASCI foram lidos para as mães e elas escolhiam a melhor resposta de cada item e se surgissem dúvidas, os pesquisadores esclareciam. O PEDI-CAT exigiu a instalação de um software em um dos computadores da instituição. Os pesquisadores também leram os itens do PEDI-CAT e conforme a resposta da mãe, preenchiam o instrumento.

\section{Análise estatística}

Os dados coletados foram inicialmente plotados em uma planilha com a utilização do software Excel (2016) e posteriormente analisados com 0 auxílio do pacote estatístico SPSS $(26,0)$. A caracterização do perfil sociodemográfico das mães e clínico das crianças foi realizada por meio de frequência absoluta $(n)$ e relativa (\%) para as variáveis categóricas; média e desvio padrão, mínimo e máximo para as variáveis contínuas. Neste estudo foram aplicados testes e técnicas estatísticas não 
paramétricas, conforme verificado por meio do teste de normalidade de Shapiro-Wilk. A análise de dados omissos foi feita a partir da estimativa das médias (EM). A correlação de Spearman foi utilizada a fim de avaliar a relação entre os escores do PEDI-CAT com a sobrecarga das mães. Em todas as análises o nível de significância adotado foi de $5 \%$ $(p<0,05)$.

\section{RESULTADOS}

A amostra foi composta por 32 crianças com SCZV e suas mães. A média de idade das crianças no momento da

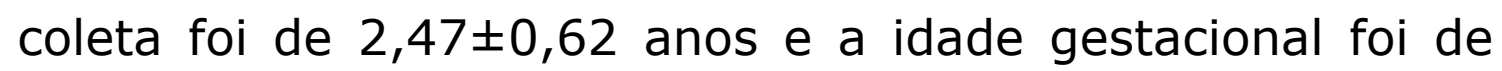
$37,86 \pm 2,01$ semanas. As mães tiveram média de idade de $30,06 \pm 6,76$ anos. Quanto ao perfil sociodemográfico das mães, todas apresentaram renda familiar de até 3 saláriosmínimos e mais de $80 \%$ recebiam algum tipo de benefício. A maioria das mães utilizava transporte que não fosse próprio, mas possuía casa própria. Apenas 4 mães (12,5\%) estavam realizando algum tratamento psicológico e $68,8 \%$ das mães possuíam até 2 filhos (Tabela 1 ).

A Tabela 2 mostra a caracterização dos aspectos clínicos das crianças com SCZV. A maioria era do sexo feminino $(65,6 \%)$, nascida a termo $(71,9 \%)$, com microcefalia $(78,2 \%)$ e todas não andavam. As crises convulsivas estavam presentes em mais da metade das crianças, assim como as alterações visuais. 
Tabela 1. Caracterização do perfil sociodemográfico das mães.

\begin{tabular}{|c|c|c|}
\hline & $\mathbf{N}$ & $\%$ \\
\hline \multicolumn{3}{|l|}{ Situação conjugal } \\
\hline Casada & 15 & 46,9 \\
\hline Solteira & 17 & 53,1 \\
\hline \multicolumn{3}{|l|}{ Atividade de lazer } \\
\hline Não & 17 & 53,1 \\
\hline Sim & 15 & 46,9 \\
\hline \multicolumn{3}{|l|}{ Renda Familiar } \\
\hline 1 a 1,5 salários & 17 & 53,1 \\
\hline 2 a 3 salários & 15 & 46,9 \\
\hline \multicolumn{3}{|l|}{ Transporte } \\
\hline Próprio & 13 & 40,6 \\
\hline Outro & 19 & 59,4 \\
\hline \multicolumn{3}{|l|}{ Residência } \\
\hline Alugada & 10 & 31,3 \\
\hline Cedida & 5 & 15,6 \\
\hline Própria & 17 & 53,1 \\
\hline \multicolumn{3}{|l|}{ Plano de Saúde } \\
\hline Não & 22 & 68,8 \\
\hline Sim & 10 & 31,3 \\
\hline \multicolumn{3}{|l|}{ Recebe benefício } \\
\hline Não & 6 & 18,8 \\
\hline Sim & 26 & 81,3 \\
\hline \multicolumn{3}{|c|}{ Em tratamento psicológico } \\
\hline Não & 28 & 87,5 \\
\hline Sim & 4 & 12,5 \\
\hline \multicolumn{3}{|l|}{ No de Filhos } \\
\hline 1 & 10 & 31,3 \\
\hline 2 & 12 & 37,5 \\
\hline 3 a 5 & 10 & 31,3 \\
\hline
\end{tabular}

A espasticidade foi o sinal clínico de maior destaque, com acometimento em $90 \%$ das crianças. Praticamente todas as crianças do estudo estavam em atendimento individualizado de fisioterapia $(96,9 \%)$ e tiveram grande adesão também na fonoaudiologia e terapia ocupacional, ambas com $87,5 \%$. 
Tabela 2. Caracterização dos aspectos clínicos das crianças com SCZV.

\begin{tabular}{|c|c|c|}
\hline & $\mathbf{N}$ & $\%$ \\
\hline \multicolumn{3}{|l|}{ Sexo } \\
\hline Feminino & 21 & 65,6 \\
\hline Masculino & 11 & 34,4 \\
\hline \multicolumn{3}{|l|}{ Idade gestacional } \\
\hline Prematuro & 6 & 18,8 \\
\hline Termo & 23 & 71,9 \\
\hline Não informado & 3 & 9,4 \\
\hline \multicolumn{3}{|c|}{ Classificação da Microcefalia } \\
\hline Ausente & 3 & 9,4 \\
\hline Grave & 19 & 59,4 \\
\hline Leve & 6 & 18,8 \\
\hline Não informado & 4 & 12,5 \\
\hline \multicolumn{3}{|l|}{ Anda } \\
\hline Não & 32 & 100,0 \\
\hline Sim & 0 & 0,0 \\
\hline \multicolumn{3}{|l|}{ Crise convulsiva } \\
\hline Não & 11 & 34,4 \\
\hline Sim & 18 & 56,3 \\
\hline \multicolumn{3}{|l|}{ Espasticidade } \\
\hline Não & 1 & 3,1 \\
\hline Sim & 29 & 90,6 \\
\hline Não informado & 2 & 6,3 \\
\hline \multicolumn{3}{|l|}{ Irritabilidade } \\
\hline Não & 9 & 28,1 \\
\hline Sim & 15 & 46,9 \\
\hline Não informado & 8 & 25,0 \\
\hline \multicolumn{3}{|l|}{ Alterações visuais } \\
\hline Não & 6 & 18,8 \\
\hline Sim & 23 & 71,9 \\
\hline Não informado & 3 & 9,4 \\
\hline \multicolumn{3}{|c|}{ Alterações auditivas } \\
\hline Não & 24 & 75,0 \\
\hline Sim & 4 & 12,5 \\
\hline Não informado & 4 & 12,5 \\
\hline
\end{tabular}

$\mathrm{n}=$ frequência absoluta; $\%$ = frequência relativa

A Tabela 3 evidencia os resultados das habilidades funcionais, mensurados por meio do PEDI-CAT. Quanto ao escore contínuo o melhor desempenho foi no domínio Social/Cognitivo $(45,5 \%)$ e o pior no domínio Atividade Diária $(36,62 \%)$. No escore-T as crianças apresentaram 
valores abaixo da média esperada pela idade em todos os domínios, sendo a Mobilidade o de maior comprometimento $(13,17 \%)$.

Tabela 3. Estatísticas descritivas do PEDI-CAT.

\begin{tabular}{cccccc}
\hline & Média & $\begin{array}{c}\text { Desvio } \\
\text { padrão }\end{array}$ & Mediana & Mínimo & Máximo \\
\hline PEDI-CAT & & & & & \\
\hline Atividade diária (Contínuo) & 36,62 & 2,82 & 36,62 & 30,00 & 41,00 \\
\hline Mobilidade (Contínuo) & 42,55 & 3,86 & 42,00 & 35,00 & 53,00 \\
\hline Social/Cognitivo (Contínuo) & 45,55 & 5,57 & 45,55 & 34,00 & 55,00 \\
\hline Atividade diária (Escore-T) & 25,31 & 8,41 & 25,31 & 10,00 & 41,00 \\
\hline Mobilidade (Escore-T) & 13,17 & 3,79 & 10,50 & 10,00 & 22,00 \\
\hline Social/Cognitivo (Escore-T) & 24,83 & 11,82 & 24,83 & 10,00 & 52,00 \\
\hline
\end{tabular}

Os resultados do índice de sobrecarga das mães, mensurado por meio do QASCI, são descritos na Tabela 4. Nela são apresentados os valores por domínio e a pontuação final. O domínio de maior sobrecarga foi a financeira e o de menor sobrecarga foi a Satisfação com o papel do familiar.

De acordo com a média do escore total, as mães apresentaram sobrecarga moderada (44,70\%). Nenhuma das mães foram classificadas dentro de valores para baixa ou extrema sobrecarga, sendo evidenciada sobrecarga moderada ou elevada, com $62,5 \%$ e $37,5 \%$, respectivamente.

A relação do PEDI-CAT com os domínios do QASCI, bem como com o seu escore total são apresentados na Tabela 5. 
Tabela 4. Estatísticas descritivas do QASCI.

\begin{tabular}{cccccc}
\hline & Média & $\begin{array}{c}\text { Desvio } \\
\text { padrão }\end{array}$ & Mediana & Mínimo & Máximo \\
\hline QASCI & & & & & \\
\hline Sobrecarga Emocional & 48,75 & 21,81 & 50,00 & 20,00 & 90,00 \\
\hline $\begin{array}{c}\text { Implicações na Vida } \\
\text { Pessoal }\end{array}$ & 48,35 & 14,81 & 45,45 & 20,00 & 74,55 \\
\hline Sobrecarga Financeira & 70,63 & 24,09 & 70,00 & 20,00 & 100,00 \\
\hline Reação às exigências & 42,13 & 22,44 & 38,00 & 20,00 & 100,00 \\
\hline $\begin{array}{c}\text { Mecanismos de Eficácia e } \\
\text { Controle }\end{array}$ & 45,00 & 18,32 & 46,67 & 20,00 & 86,67 \\
\hline Suporte Familiar & 37,19 & 19,71 & 40,00 & 20,00 & 100,00 \\
\hline $\begin{array}{c}\text { Satisfação com o Papel e } \\
\text { com o Familiar }\end{array}$ & 20,88 & 3,00 & 20,00 & 20,00 & 36,00 \\
\hline Escore total & 44,70 & 11,64 & 44,91 & 24,86 & 68,20 \\
\hline
\end{tabular}

Todas as correlações encontradas foram moderadas. Correlação positiva, ou seja, diretamente proporcional foi observada entre o domínio reação às exigências do QASCI e os domínios Atividade diária (escore contínuo e escore-T) e Mobilidade (escore-T) do PEDI-CAT. O domínio mecanismos de eficácia e controle foi correlacionado positivamente com todos os domínios do PEDI-CAT por meio do escore-T.

O domínio satisfação com o papel e o com o familiar, foi correlacionado de forma negativa com os domínios Atividade diária e Mobilidade (escore contínuo).

Na pontuação final do QASCI, houve correlação positiva com o domínio Mobilidade (escore-T). Esse resultado mostra que, quanto maior as habilidades funcionais das crianças, no que diz respeito a mobilidade, maior a sobrecarga de suas mães. 
Tabela 5. Correlação entre os escores do PEDI-CAT com os domínios e o escore total do QASCI.

\begin{tabular}{|c|c|c|c|c|c|c|}
\hline & \multicolumn{3}{|c|}{ Escore contínuo } & \multicolumn{3}{|c|}{ Escore-T } \\
\hline & $\begin{array}{l}\text { Atividade } \\
\text { diária }\end{array}$ & Mobilidade & $\begin{array}{c}\text { Social/ } \\
\text { Cognitivo }\end{array}$ & $\begin{array}{l}\text { Atividade } \\
\text { diária }\end{array}$ & Mobilidade & $\begin{array}{l}\text { Social/ } \\
\text { Cognitivo }\end{array}$ \\
\hline \multicolumn{7}{|l|}{ QASCI } \\
\hline $\begin{array}{c}\text { Sobrecarga } \\
\text { Emocional }\end{array}$ & $\begin{array}{l}\rho=-0,02 \\
p=0,92\end{array}$ & $\begin{array}{l}\rho=0,17 \\
p=0,37\end{array}$ & $\begin{array}{l}\rho=0,05 \\
p=0,81\end{array}$ & $\begin{array}{l}\rho=0,13 \\
p=0,46\end{array}$ & $\begin{array}{l}\rho=0,17 \\
p=0,35\end{array}$ & $\begin{array}{l}\rho=0,20 \\
p=0,27\end{array}$ \\
\hline $\begin{array}{c}\text { Implicações } \\
\text { na Vida } \\
\text { Pessoal } \\
\end{array}$ & $\begin{array}{l}\rho=0,16 \\
p=0,40\end{array}$ & $\begin{array}{l}\rho=0,26 \\
p=0,16\end{array}$ & $\begin{array}{l}\rho=0,20 \\
p=0,28\end{array}$ & $\begin{array}{l}\rho=0,19 \\
p=0,30\end{array}$ & $\begin{array}{l}\rho=0,21 \\
p=0,25\end{array}$ & $\begin{array}{l}\rho=0,26 \\
p=0,15\end{array}$ \\
\hline $\begin{array}{c}\text { Sobrecarga } \\
\text { Financeira }\end{array}$ & $\begin{array}{l}\rho=0,13 \\
p=0,46\end{array}$ & $\begin{array}{l}\rho=0,07 \\
p=0,69\end{array}$ & $\begin{array}{l}\rho=0,07 \\
p=0,71\end{array}$ & $\begin{array}{l}\rho=0,24 \\
p=0,18\end{array}$ & $\begin{array}{l}\rho=0,10 \\
p=0,57\end{array}$ & $\begin{array}{l}\rho=0,21 \\
p=0,25\end{array}$ \\
\hline $\begin{array}{l}\text { Reação às } \\
\text { exigências }\end{array}$ & $\begin{array}{l}\rho=0,42 \\
p=0,02\end{array}$ & $\begin{array}{l}\rho=0,27 \\
p=0,13\end{array}$ & $\begin{array}{l}\rho=0,12 \\
p=0,50\end{array}$ & $\begin{array}{l}\rho=0,36 \\
p=0,04\end{array}$ & $\begin{array}{l}\rho=0,46 \\
p=0,01\end{array}$ & $\begin{array}{l}\rho=0,24 \\
p=0,18\end{array}$ \\
\hline $\begin{array}{c}\text { Mecanismos } \\
\text { de Eficácia e } \\
\text { Controle }\end{array}$ & $\begin{array}{l}\rho=0,15 \\
p=0,41\end{array}$ & $\begin{array}{l}\rho=0,29 \\
p=0,11\end{array}$ & $\begin{array}{l}\rho=0,13 \\
p=0,49\end{array}$ & $\begin{array}{l}p=0,34 \\
p=0,02\end{array}$ & $\begin{array}{l}\rho=0,38 \\
p=0,03\end{array}$ & $\begin{array}{l}p=0,39 \\
p=0,03\end{array}$ \\
\hline $\begin{array}{l}\text { Suporte } \\
\text { Familiar }\end{array}$ & $\begin{array}{l}\rho=-0,18 \\
p=0,32\end{array}$ & $\begin{array}{l}\rho=0,09 \\
p=0,63\end{array}$ & $\begin{array}{l}\rho=0,06 \\
p=0,73\end{array}$ & $\begin{array}{l}\rho=-0,08 \\
p=0,66\end{array}$ & $\begin{array}{l}\rho=0,19 \\
p=0,30\end{array}$ & $\begin{array}{l}\rho=0,18 \\
p=0,33\end{array}$ \\
\hline $\begin{array}{c}\text { Satisfação } \\
\text { com o Papel } \\
\text { e com o } \\
\text { Familiar } \\
\end{array}$ & $\begin{array}{c}\rho=-0,45 \\
p=0,01\end{array}$ & $\begin{array}{c}p=-0,48 \\
p=0,01\end{array}$ & $\begin{array}{l}\rho=-0,27 \\
p=0,14\end{array}$ & $\begin{array}{l}\rho=-0,21 \\
p=0,25\end{array}$ & $\begin{array}{l}\rho=-0,19 \\
p=0,29\end{array}$ & $\begin{array}{l}\rho=-0,12 \\
p=0,51\end{array}$ \\
\hline Escore total & $\begin{array}{l}\rho=0,14 \\
p=0,45\end{array}$ & $\begin{array}{l}\rho=0,27 \\
p=0,14\end{array}$ & $\begin{array}{l}\rho=0,12 \\
p=0,52\end{array}$ & $\begin{array}{l}\rho=0,27 \\
p=0,13\end{array}$ & $\begin{array}{l}\rho=0,36 \\
p=0,04\end{array}$ & $\begin{array}{l}\rho=0,33 \\
p=0,06\end{array}$ \\
\hline
\end{tabular}

$\rho=$ correlação de Spearman

\section{DISCUSSÃO}

Os achados desse estudo apontam que as habilidades funcionais das crianças com SCZV são gravemente afetadas em todos os domínios do PEDI-CAT, com pior desempenho na mobilidade (de acordo com o escore-T). Dados que corroboram com a pesquisa de Rodrigues ${ }^{16}$ que também aplicou o PEDI-CAT em crianças com SCZV. Ele avaliou 48 crianças com média de idade de 2,75 anos e mais de $90 \%$ apresentaram valores abaixo da média esperada para a idade em todos os domínios. Embora a média do escore-T 
entre os domínios tenha sido bastante próxima, a mobilidade também obteve menor média.

O pior desempenho na mobilidade, também, foi apontado por Ferreira et al. ${ }^{17}$ que utilizaram a Classificação Internacional de Funcionalidade Incapacidade e Saúde para Crianças e Jovens (CIF-CJ) em 34 crianças com SCZV com idade próxima a 2 anos. A categoria Atividades e Participação foi fortemente impactada e mais uma vez a mobilidade representada pelo pior desempenho. Logo sugere-se que a mobilidade é o domínio de maior comprometimento em crianças com SCZV.

O PEDI-CAT foi considerado como instrumento válido para medida de atividade e participação da CIF-CJ. Dentro do PEDI-CAT, o domínio mobilidade obteve correspondência com os capítulos de mobilidade da CIF-CJ, com $94 \%$ de concordância entre os examinadores ${ }^{18}$. Portanto, esses resultados reiteram a necessidade de medidas de intervenção quanto a mobilidade das crianças com SCZV ${ }^{19}$.

Neste estudo todas as mães apresentaram nível de sobrecarga moderada ou elevada. A sobrecarga financeira foi o domínio de maior comprometimento nas mães avaliadas. A mãe muitas vezes tende a abandonar a carreira profissional para dedicação exclusiva à criança. Os gastos com crianças com alguma deficiência dificultam ainda mais a condição financeira das famílias. E no caso das crianças com SCZV essa situação agrava-se, pois já são famílias vulneráveis financeiramente. Essa situação sinaliza a 
necessidade de maior suporte social e incremento das políticas públicas que auxiliem na dinâmica das famílias ${ }^{6,20,21}$.

Essa sobrecarga pode repercutir em vários aspectos, sendo que a sobrecarga apresenta correlação de moderada a forte para a depressão e ansiedade ${ }^{22}$, o que demostra que a saúde emocional dessas mães estava abalada. A saúde emocional dessas mães por vezes é discutida de forma qualitativa e generalizada, revelando a necessidade de estudos mais precisos ${ }^{23}$.

Um estudo realizado no nordeste brasileiro evidenciou escores significativamente maiores de depressão em mães de crianças com SCZV entre 15 e 26 meses, do que aquelas com filhos com desenvolvimento típico. Por vezes a mãe vêse em condições de sofrimento, pois destina um grande tempo de sua rotina com a assistência prestada, e todos os esforços não repercutem em ganhos motoras que elas almejam ${ }^{24}$.

Kuper et al. ${ }^{6}$ avaliaram mães de crianças com SCZV e crianças com desenvolvimento motor típico. As crianças de ambos os grupos tinham em sua maioria idade superior a 12 meses. Foi encontrada maior predisposição ao desenvolvimento de depressão, ansiedade e estresse nas mães de crianças com SCZV, sendo o baixo suporte social um fator de possível influência.

Em nosso estudo, o domínio satisfação com o papel e com o familiar do QASCI que representa o quanto a mãe sente-se bem e especial em cuidar do seu filho, obteve baixa sobrecarga, além de ser o menos afetado. Isto sugere que a 
satisfação e o vínculo das mães com essas crianças pode ser um fator positivo de enfrentamento ${ }^{25}$.

No que diz respeito a relação entre as habilidades funcionais e sobrecarga das mães de crianças com SCZV, não foram encontrados outros estudos que investigaram esta relação. Além disso, outras pesquisas com temática similar possuíam objetivos e metodologias diferentes, inviabilizando correlacionar seus resultados com o presente estudo.

No presente estudo, houve relação entre alguns domínios da sobrecarga e as habilidades funcionais das crianças. No entanto, ao analisar o escore total de sobrecarga a única correlação foi com o domínio mobilidade do PEDI-CAT (escore-T).

Nas crianças com pior desempenho nos domínios mobilidade e atividade diária do PEDI-CAT, ou seja, mais graves, a sobrecarga das mães foi menor quanto aos domínios de reação às exigências e mecanismo de eficácia e controle do QASCI. Essas mesmas mães tiveram maior sobrecarga no domínio satisfação com o papel e com o familiar do QASCI.

O domínio reação às exigências diz respeito ao quanto o filho solicita a mãe e a falta de privacidade decorrente disso. Já o domínio mecanismo de eficácia e controle descreve o quanto a mãe consegue fazer as atividades que necessita, a sua capacidade e conhecimento para o cuidado do filho. E o domínio satisfação com o papel e com o familiar retrata o quanto a mãe sente-se bem e especial em cuidar do filho ${ }^{25}$. 
As crianças mais graves tiveram mães mais adaptadas para o cuidado. Elas também sentiam que tinham conhecimento e capacidade para o manejo com os seus filhos, e que este filho não a solicitava com frequência. Em contrapartida, essas mesmas mães não se sentiam tão especiais e satisfeitas em cuidar do seu filho em comparação com mães de crianças com maior habilidade funcional.

Em outro estudo, a maior dificuldade apresentada por mães de crianças com Paralisia Cerebral (PC) grave foi lidar com as limitações funcionais dos filhos, expondo-as a sobrecarga. Além disso as mães apresentaram sentimentos negativos, como tristeza e decepção ao compararem os seus filhos com os demais, e por almejarem ganhos funcionais não adquiridos 26 .

Intervenções da equipe multiprofissional precisam ser direcionadas as mães de crianças com SCZV, quer sejam condutas de orientação e capacitação para as mães de crianças com melhor habilidade funcional, ou maior suporte psicológico para aquelas com filhos mais graves.

A amostra deste estudo mostrou-se homogênea quanto ao comprometimento das habilidades funcionais. A média do escore-T foi abaixo do esperado para a faixa etária em todos os domínios do PEDI-CAT e nenhuma criança obteve escore satisfatório no domínio mobilidade. Portanto, mesmo as crianças que obtiveram melhor desempenho, ainda assim são dependentes das mães para a realização das suas atividades diárias e mobilidade completa. 
Essa amostra homogênea limita a possível correlação entre crianças com melhores habilidades funcionais e a maior sobrecarga de suas mães. Uma possível explicação seria o fato dessa criança com mobilidade menos prejudicada conseguir pelo menos iniciar um movimento. Com isso, a mãe poderia ter maior sobrecarga ao considerar essa situação psicologicamente difícil, preocupando-se com possíveis intercorrências, como quedas de uma cama, berço e afins.

No entanto, essa relação entre mobilidade e sobrecarga tem sido divergente entre os estudos com crianças com PC. Um estudo utilizou o GMFCS em diferentes faixas etárias e relacionou maior comprometimento motor com maior sobrecarga das mães ${ }^{27}$. Resultado divergente foi encontrado no estudo de Camargos et al. ${ }^{28}$, o qual verificou-se que crianças com menor comprometimento motor possuíam cuidadores com maior sobrecarga. Já no estudo de Barbosa et al. ${ }^{29}$, o comprometimento motor da criança com PC não apresentou relação com o estresse e a sobrecarga do cuidador.

Sabe-se que a chegada de um filho com deficiência altera a rotina familiar, e que muitas vezes ocorrerão situações de oposição ao lidar com a criança, e a mãe necessitará adaptar sua rotina para satisfazer as necessidades da criança $\mathrm{e}$ as suas. Independente da gravidade motora das crianças com deficiência, seus cuidadores experimentam esses enfrentamentos ${ }^{30}$. 
A nossa pesquisa é relevante, pois analisou as habilidades funcionais das crianças com SCZV com ênfase nas atividades e participação, e a sobrecarga das mães por meio de um instrumento validado para o Brasil e com o uso autorizado para essa população específica. Também é relevante porque abre espaço para a investigação de demais fatores intervenientes na saúde emocional das mães.

Ao detectarmos a mobilidade como o maior comprometimento investigado, pretende-se sinalizar a importância das intervenções focadas nos fatores ambientais modificáveis, visto que essas crianças não irão adquirir mobilidade independente ${ }^{18}$.

Como limitações e dificuldades do estudo, podemos citar o pequeno tamanho amostral, além da ausência de um grupo de crianças com infecções congênitas por outras causas para comparação e análise dos resultados.

\section{CONCLUSÃO}

As habilidades funcionais das crianças com SCZV desse estudo são gravemente impactadas. Além disso, suas mães apresentaram nível de sobrecarga moderada ou elevada. Quanto pior as habilidades funcionais (mobilidade e atividade diária) das crianças, menor a sobrecarga das mães nos domínios reação às exigências e mecanismos de eficácia e controle, e maior sobrecarga no domínio satisfação com o papel e com o familiar. Além disso, o escore total de sobrecarga parece estar relacionado com as habilidades funcionais da criança, sendo que quanto melhor as 
habilidades funcionais no que diz respeito à mobilidade, maior a sobrecarga das mães.

\section{REFERÊNCIAS}

1. Melo A, Gama GL, Da Silva Júnior RA, De Assunção PL, Tavares JS, Da Silva MB, et al. Motor function in children with congenital Zika syndrome. Dev Med Child Neurol 2020;62:221-16. https://dx.doi.org/10.1111/dmcn.14227

2.Teixeira GA, Dantas NAD, Carvalho GAFL, Silva AN, Lira ALBC, Enders $B C$. Análise do conceito síndrome congênita pelo Zika vírus. Ciênc Saúde Coletiva 2020;25:567-74. http://dx.doi.org/10.1590/141381232020252.30002017

3.Pereira HVFS, Dos Santos SP, Amâncio APRL, de Oliveira-Szejnfeld PS, Flor EO, de Sales Tavares J, et al. Neurological outcomes of congenital Zika syndrome in toddlers and preschoolers: a case series. Lancet Child Adolesc Health 2020;4:378-87. https://dx.doi.org/10.1016/S2352-4642(20)30041-9

4.Frota LMDCP, Sampaio RF, Miranda JL, Brasil RMC, Gontijo APB, Mambrini JVM, et al. Children with congenital Zika syndrome: symptoms, comorbidities and gross motor development at 24 months of age. Heliyon 2020;15:1-8.

https://dx.doi.org/10.1016/j.heliyon.2020.e04130

5.Saleh M, Almasri NA, Malkawi SH, Abu-Dahab S. Associations between impairments and activity limitations components of the international classification of functioning and the gross motor function and subtypes of children with cerebral palsy. J Phys Ther Sci 2019;31:299-305. https://dx.doi.org/10.1589/jpts.31.299

6.Kuper $\mathrm{H}$, Lopes Moreira ME, Barreto de Araújo TV, Valongueiro S, Fernandes S, Pinto $M$, et al. The association of depression, anxiety, and stress with caring for a child with Congenital Zika Syndrome in Brazil; Results of a cross-sectional study. PLoS Negl Trop Dis 2019;13:1-12. https://dx.doi.org/10.1371/journal.pntd.0007768

7.Ozkan Y. Child's quality of life and mother's burden in spastic cerebral palsy: a topographical classification perspective. J Int Med Res 2018;46:3131-7. https://dx.doi.org/10.1177/0300060518772758

8.Brunoni D, Blascovi-Assis SM, Osório AAC, Seabra AG, Amato CAH, Teixeira MCTV, et al. Microcefalia e outras manifestações relacionadas ao vírus Zika: impacto nas crianças, nas famílias e nas equipes de saúde. Ciênc Saúde Coletiva 2016;21:3297-302. https://dx.doi.org/10.1590/1413-812320152110.16832016

9. Monteiro EA, Mazin SC, Dantas RAS. Questionário de Avaliação da Sobrecarga do Cuidador Informal: validação para o Brasil. Rev Bras Enferm 2015;6:421-8. $\quad$ http://dx.doi.org/10.1590/00347167.2015680307i

10.Mancini MC, Coster WJ, Amaral MF, Avelar BS, Freitas R, Sampaio RF. New version of the Pediatric Evaluation of Disability Inventory 
(PEDI-CAT): translation, cultural adaptation to Brazil and analyses of psychometric properties. Braz J Phys Ther 2016;20:56170. https://dx.doi.org/10.1590/bjpt-rbf.2014.0166

11.Martins T, Ribeiro, JP, Garrett C. Estudo de validação do Questionário de Avaliação da Sobrecarga para Cuidadores Informais. Psicol Saúde Doenças 2003;4:131-48. http://hdl.handle.net/10400.12/1050

12. Martins T, Ribeiro JP, Garrett C. Questionário de Avaliação de Sobrecarga do Cuidador Informal (QASCI): Reavaliação das Propriedades Psicométricas. Referência 2004;11:17-31. https://rr.esenfc.pt/rr/index.php? module $=$ rr\&target $=$ publicationDetail s\&pesquisa $=$ \&id artigo $=35 \&$ id revista $=5 \&$ id edicao $=10$

13.Gomes SDM. Avaliação dos Cuidadores de Doentes com Demência (dissertação). Covilhã: Universidade da Beira Interior, 2011, 70p. http://hdl.handle.net/10400.6/950

14.Shore BJ, Allar BG, Miller PE, Matheney TH, Snyder BD, FragalaPinkham M. Measuring the Reliability and Construct Validity of the Pediatric Evaluation of Disability Inventory-Computer Adaptive Test (PEDI-CAT) in Children With Cerebral Palsy. Arch Phys Med Rehabil 2019;100:45-51. https://dx.doi.org/10.1016/j.apmr.2018.07.427

15. Haley SM, Coster WJ, Dumas HM, Fragala-Pinkham MA, Kramer J, $\mathrm{Ni} \mathrm{P}$, et al. Accuracy and precision of the Pediatric Evaluation of Disability Inventory computer-adaptive tests (PEDI-CAT). Dev Med Child Neurol 2011;53:1100-6. htps://dx.doi.org/10.1111/j.14698749.2011.04107.X

16.Rodrigues ASR. Achados Clínicos, de neuroimagem e videoencefalograma e a relação com a funcionalidade de crianças com Síndrome Congênita pelo Vírus Zika (dissertação). Fortaleza: Universidade Federal do Ceará, 2019, 155p. http://www.repositorio.ufc.br/handle/riufc/46966

17.Ferreira HNC, Schiariti V, Regalado ICR, Sousa KG, Pereira SA, Fechine CPNDS, et al. Functioning and Disability Profile of Children with Microcephaly Associated with Congenital Zika Virus Infection. Int J Environ Res Public Health 2018;15:1107. https://dx.doi.org/10.3390/ijerph15061107

18.Thompson SV, Cech DJ, Cahill SM, Krzak JJ. Linking the Pediatric Evaluation of Disability Inventory-Computer Adaptive Test (PEDI-CAT) to the International Classification of Function. Pediatr Phys Ther 2018;30:113-8. https://dx.doi.org/10.1097/PEP.0000000000000483 19.Longo E, De Campos AC, Spinola BA, Lima NCDL, Leite GCM, Corsi C, et al. Go Zika Go: A Feasibility Protocol of a Modified Ride-on Car Intervention for Children with Congenital Zika Syndrome in Brazil. Int J Environ Res Public Health 2020;17:6875. https://dx.doi.org/10.3390/ijerph17186875

20.Basaran A, Karadavut KI, Uneri SO, Balbaloglu O, Atasoy N. The effect of having a children with cerebral palsy on quality of life, burnout, depression and anxiety scores: a comparative study. Eur J Phys Rehabil Med 2013;49:815-22. https://www. minervamedica.it/en/getfreepdf/SHIRaWhhTzZ6NTNnZF 
NZMDF2WkIWZFo0K1hYMXNybkNsa3UrVjU1aEhoVzFxdFhpR2FKK1gyc kYOUIJCZ2dkWg\%253D\%253D/R33Y2013N06A0815.pdf

21.Rodrigues SA, Fontanella BJB, de Avó LRS, Germano CMR, Melo DG. A qualitative study about quality of life in Brazilian families with children who have severe or profound intellectual disability. J Appl Res Intellect

Disabil

2019;32:413-26.

https://dx.doi.org/10.1111/jar.12539

22. Wijesinghe CJ, Cunningham N, Fonseka P, Hewage CG, Østbye T. Factors associated with caregiver burden among caregivers of children with cerebral palsy in Sri Lanka. Asia Pac J Public Health 2015;27:8595. https://dx.doi.org/10.1177/1010539514548756

23. Freitas PSS, Soares GB, Mocelin HJS, Lamonato LCXL, Sales CMM, Linde-Arias $A R$, et al. How do mothers feel? Life with children with congenital Zika syndrome. Gynecol Obstetric 2020;148:20-8. https://dx.doi.org/10.1002/ijgo.13044

24.Kotzky K, Allen JE, Robinson LR, Satterfield-Nash A, Bertolli J, Smith $\mathrm{C}$, et al. Depressive Symptoms and Care Demands Among Primary Caregivers of Young Children with Evidence of Congenital Zika Virus Infection in Brazil. J Dev Behav Pediatr 2019;40:344-53. https://dx.doi.org/10.1097/DBP.0000000000000666

25.Veríssimo TCRA. Evolução do nível de sobrecarga de mães de crianças com Síndrome Congênita do Zika Vírus (dissertação). Goiânia: Pontifícia Universidade Católica de Goiás, 2019, 83p. http://tede2.pucgoias.edu.br:8080/handle/tede/4258

26. Ribeiro MFM, Vandenberghe L, Prudente COM, Vila VSC, Porto CC. Paralisia cerebral: faixa etária e gravidade do comprometimento do filho modificam o estresse e o enfrentamento materno. Ciênc. saúde coletiva 2016;21:3203-12. https://dx.doi.org/10.1590/1413812320152110.17352016

27.Yilmaz H, Erkin G, Nalbant L. Depression and anxiety levels in mothers of children with cerebral palsy: a controlled study. Eur J Phys Rehabil Med 2013;49:823-7. https://www.minervamedica.it/en/getfreepdf/eFc2ZTVuaklINkVIc0diQ kdKeEZzNnRzYW9QdExGMDRhQ1VzbHVGZXpmd0xXUmZiVIJnOWFmR isXRkFNbG9XSQ\%253D\%253D/R33Y2013N06A0823.pdf

28.Camargos ACR, Lacerda TTB, Viana SO, Pinto LRA, Fonseca MLS. Avaliação da sobrecarga do cuidador de crianças com paralisia cerebral através da escala Burden Interview. Rev Bras Saude Mater Infant 2009;9:31-7.

https://dx.doi.org/10.1590/S151938292009000100004

29.Barbosa APM, Zampa TM, Iwabe C, Diz MAR. Relação da Sobrecarga do Cuidador, Perfil Funcional e Qualidade de Vida em Crianças com Paralisia Cerebral. Rev Neurocienc 2012;20:367-71. https://dx.doi.org/10.34024/rnc.2012.v20.8253

30.Shevell M, Oskoui M, Wood E, Kirton A, Van Rensburg E, Buckley D, et al. Family-centred health care for children with cerebral palsy. Dev Med Child Neurol 2019;61:62-8. https://dx.doi.org/10.1111/dmcn.14053 